\title{
Detección de norovirus en niños con diarrea adquirida en la comunidad o nosocomial en el Hospital Guillermo Grant Benavente de Concepción, Chile
}

\author{
Sonia Montenegro, Susana Pineda, Isabel Enríquez, Nancy Enríquez, Nancy Rivera y Carolina Delgado
}

Universidad de Concepción, Concepción, Chile. Facultad de Medicina (SM, NR, CD) . Hospital Guillermo Grant Benavente, Concepción, Chile. (SP, IE, NE).

Financiamiento: Proyecto DIUC No 207.86.06-1

Recibido: 27 de agosto de 2013 Aceptado: 3 de junio de 2014

Correspondencia a: Sonia Montenegro Heredia labpatologiamolecular@gmail.com

\section{Norovirus detection in children with community-acquired or nosocomial diarrhea at Guillermo Grant Benavente Hospital in Concepción, Chile}

Introduction: Norovirus (NoV) are RNA viruses highly contagious, stable in the environment, genetically variable, and the most common cause of viral sporadic acute gastroenteritis worldwide. This is the first study carried out in Concepcion, Chile, to investigate the presence of $\mathrm{NoV}$ as an etiologic agent of viral diarrheas in hospitalized children. Objective. To detect the presence and genogroup of NoV in children with diarrhea and to compare it with rotavirus (RV) and adenovirus (AdV). Material and Methods: A one year descriptive, prospective study in children 0-14 years old. A single diarrheic stool sample per patient was analyzed for the presence of NoV, RV and AdV. Clinical data were unknown at the moment of sampling. Real time RT-PCR with Taqman ${ }^{\mathrm{TM}}$ probes for $\mathrm{NoV}$ and the immunocromatography VIKIA ${ }^{\mathrm{TM}}$ kit for RoV / AV detection were used. Results: Infection for $\operatorname{NoV}(25.5 \%)$ was significantly higher than for RV (15.9\%) and AdV (6.2\%). It was even greater in infants younger than 2yr. old (n: 103): NoV 34\%, RV 17.5\%, AdV 7.8\%. Children 2-4 yr. old had 11.8\% infection of NoV and RV. Children older than 4, only had 12\% RV and 4\% AdV. Children hospitalized for diarrhea (n: 92) had: $21.7 \%$ of both NoV and RV, and 7.6\% AdV; whereas children hospitalized for other causes (n: 53 ) had 32.1\% NoV,5.7\% RV and 3.8\% AV. The proportion of infection due to NoV was significantly higher in males (31.5\%) than in females (19.4\%). The average frequency during the year was higher for NoV (30.3\%) than for RV (14.7\%) except in summer. Conclusion: The presence of NoV was higher than RV in children with diarrhea. NoV infection showed defined characteristics regarding age, gender, seasonal occurrence and nosocomial transmission that are important epidemiological features.

Key words: Norovirus, acute gastroenteritis, rotavirus.

Palabras clave: Norovirus, gastroenteritis, rotavirus.

\section{Introducción}

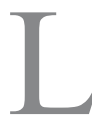
as diarreas son una importante causa de morbilidad y mortalidad en niños bajo 5 años de edad ${ }^{1,2}$ y su principal etiología es viral ${ }^{3-5}$. Los norovirus ( $\mathrm{NoV}$ ) son patogenos emergentes, que causan hospitalización e infecciones nosocomiales en niños y adultos ${ }^{6-9} \mathrm{y}$ son responsables hasta de $95 \%$ de brotes de gastroenteritis viral aguda esporádica en el mundo ${ }^{10,11}$. Son considerados agentes de biodefensa categoría B por su extremada estabilidad ambiental y contagiosidad, resistencia a desinfectantes comunes y asociación a enfermedades debilitantes ${ }^{10}$. Se transmiten por agua o alimentos contaminados, o por contacto persona a persona y exposición a fómites ${ }^{3,4}$ y los brotes generalmente ocurren en comunidades semiconfinadas como hospitales, escuelas, cruceros y asilos ${ }^{5-7}$. Aunque la infección es auto-limitada, la enfermedad puede ser mucho más intensa en lactantes, ancianos e individuos inmunocomprometidos ${ }^{4,9-11}$; por esto, el diagnóstico debería ser rápido, sensible y específico, para el mejor manejo de brotes de gastroenteritis en la comunidad $y$ en hospitales ${ }^{11-13}$. La presencia de NoV ha aumentado en la última década, lo que podría deberse a un mejor diagnóstico o a la emergencia de cepas más virulentas ${ }^{10}$.

Los NoV son virus ARN de la familia Caliciviridae, de gran diversidad genética, con aparición continua de nuevas variantes genéticas, lo que dificulta su diagnósti$\mathrm{co}^{6-8}$. De los cinco genogrupos (G) de NoV, los GI, GII y GIV infectan a humanos ${ }^{4,7,9}$; el GII es el prevalente y ha causado brotes epidémicos en diferentes regiones del mundo, en tanto que el GI es de menor frecuencia y se ha encontrado principalmente asociado a brotes alimentarios y muestras ambientales ${ }^{4,11,14-15}$.

En Chile el cuadro de diarreas en niños bajo 5 años de edad es una patología de alta frecuencia y baja mortalidad $\left(0,8\right.$ por 100.000 en el año 2004) ${ }^{16}$, siendo considerado 
un problema importante de salud pública, con alta demanda de atenciones ambulatorias y ha sido asociada a desnutrición ${ }^{2,16,17}$.

En general, se considera que la principal etiología viral de diarreas en niños son los rotavirus (RV) y ha sido en Chile la etiología más frecuente ${ }^{18}$. Los calicivirus se reportaron, en comienzo, serológicamente ${ }^{19}$; en el año 2000 se introdujo el diagnóstico por biología molecular (reacción de polimerasa en cadena RPC-TR en tiempo real), encontrándose una prevalencia de $8 \%{ }^{20}$. En el 2005, un estudio efectuado en la ciudad de Santiago por Vidal y cols., reportó que $45 \%$ de 55 brotes de gastroenteritis por consumo de mariscos fueron causados por $\mathrm{NoV}^{21}$. Posteriormente, O'Ryan y cols., en un estudio llevado a cabo en niños con diarrea, encontró $18 \%$ de NoV y $26 \%$ de $\mathrm{RV}^{22}$.

En Chile, la vigilancia epidemiológica de las diarreas en niños bajo 5 años de edad, se realiza en 34 centros centinelas de todo el país ${ }^{16,17}$, programa que incluye la determinación de su etiología ${ }^{16}$.

En el año 2010, el Ministerio de Salud de Chile (MINSAL) reportó un brote de gastroenteritis aguda asociado a NoV, entre otros agentes, que afectó a más de 30 mil personas en Antofagasta ${ }^{23}$. En el año 2011 se reportó una mayor incidencia de infecciones causadas por NoV $(27,1 \%)$ que por RV $(19,6 \%)$ considerándose esta etiología viral (NoV) como la principal causa de diarrea ${ }^{17}$.

La etiología viral por RV y adenovirus (AdV) en diarreas de niños bajo 5 años de edad en la Región del Bío-Bío está monitoreada por los centros centinelas. Debido a la importancia creciente de NoV, se incorporó la detección de NoV en tres centros centinelas a partir del año $2012^{24}$.

Las infecciones asociadas a hospitales son una causa importante de morbilidad y mortalidad en los recintos hospitalarios $^{25,26}$. Se estima que en Estados Unidos de América (E.U.A), entre 20 y $40 \%$ de las infecciones nosocomiales se deben a infección cruzada, vía manipulación por personal del hospital, contaminado a su vez ,a través de contacto directo con el paciente o en forma indirecta tocando superficies contaminadas ${ }^{25,26}$. Existe evidencia que la contaminación ambiental también juega un rol en la transmisión nosocomial de NoV debido a que es un patógeno que sobrevive por prolongados periodos en el ambiente $^{10,11}$. Por esta razón, los brotes de NoV nosocomial son una de las principales causas de cierre de salas en los hospitales de países desarrollados y cuarentena de navíos de cruceros ${ }^{10}$.

Por todos estos antecedentes, consideramos importante el estudio de NoV cuyo objetivo fue determinar la presencia de NoV y los genogrupos predominantes, comparándolos con la presencia de RV y AdV en niños con diarrea hospitalizados en Concepción. Para cumplir este objetivo se utilizó el método Taqman de RPC-TR a tiempo real recomendado por el Centers for Disease Control and Prevention (CDC) de Atlanta, E.U.A. ${ }^{27}$ y que es el método actualmente utilizado por el ISP para la detección de $\mathrm{NoV}$ en Chile.

\section{Material y Métodos}

Tipo de estudio: Descriptivo, prospectivo.

Universo: Niños con diarrea, entre un mes y14 años de edad, internados en el Hospital Regional Dr. Guillermo Grant Benavente de Concepción (HGGB), procedentes de todas las comunas y provincias de la Región del BíoBío, en el período comprendido entre diciembre de 2007 y diciembre de 2008 .

Muestra: Se analizó una muestra de diarrea por paciente, que se colectó a solicitud del pediatra tratante, para análisis etiológico de RV y AdV. Se utilizó una alícuota de la misma muestra para detección de NoV. Se analizaron 145 deposiciones de 73 niños y 72 niñas.

Controles: Constituido por 57 niños sin diarrea, ajustados a edad y sexo, procedentes de salas cuna o jardines infantiles de Concepción. Muestreo intencionado.

Clasificación de los pacientes por causa de hospitalización: Pacientes hospitalizados con SDA fueron definidos como aquellos que se internaron con diarrea. Pacientes con infección nosocomial fueron considerados aquellos que se internaron por otras causas (p. ej.: cuadros respiratorios, inmunodeficiencias, leucemia, infecciones del tracto urinario, otras) y que presentaron diarrea después de tres días de ser hospitalizados.

Criterios de inclusión: Niños internados, con diarrea en el momento de la toma de muestra. Muestras derivadas al laboratorio de Microbiología del HGGB por el pediatra tratante, para pesquisa de RV y AdV. Muestras con bacteriología y parasitología negativa. Consentimiento informado autorizado por los padres, para la búsqueda de NoV en la deposición.

Criterios de exclusión: Niños hospitalizados, sin diarrea, y que la presentaron antes del tercer día de internación. Estar con tratamiento anti-diarreico y niños controles con diarrea.

Consentimiento informado: Se obtuvo el consentimiento informado de los padres y todos los procedimientos fueron aprobados por los Comités de Ética del Hospital HGGB y de la Universidad de Concepción (UDEC). 


\section{Detección de norovirus}

Procesamiento de muestras y extracción de ARN. La alícuota de muestra de diarrea de pacientes y controles para análisis de NoV se transportó en un frasco estéril al Laboratorio de Diagnóstico Clínico Molecular-UDEC con una unidad refrigerante. Se prepararon suspensiones de heces en tampón salino fosfatado (PBS) y se almacenaron a $-80^{\circ} \mathrm{C}$ hasta su uso. El ARN fue extraído con el kit QIAamp Viral RNA® de Qiagen, siguiendo las instrucciones del fabricante. El ARN obtenido se almacenó a $-80^{\circ} \mathrm{C}$ y fue utilizado dentro de los tres meses de extraído ${ }^{28}$.

Detección de norovirus por el método Taqman RPC$T R$ a tiempo real (CDC). Se utilizaron $5 \mathrm{ml}$ de $\mathrm{ARN}$ de la muestra para la síntesis de cDNA mediante el kit Qiagen One-Step RT-PCR seguida de RPC a tiempo real con sondas Taqman específicas para cada genogrupo de NoV. Utilizamos $1 \mu \mathrm{M}$ de cada partidor COG2-F y COG2-R para amplificación de NoV GII y los partidores COG-1F y COG-1R para la amplificación de NoV GI, que tienen secuencias conservadas del genoma de NoV localizadas en la unión de la región ORF1 (región polimerasa) y ORF2 (región cápside viral) ${ }^{27,29}$. La hibridación del producto de RPC se realizó con $0,1 \mu \mathrm{M}$ de cada sonda Taqman específica, Ring-2TP y Ring-1A/Ring-1B para los GII y GI, respectivamente ${ }^{27,29}$. La síntesis de cDNA se realizó a $50^{\circ} \mathrm{C}$ por $30 \mathrm{~min}$ y la amplificación de $\mathrm{NoV}$ a $95^{\circ} \mathrm{C} / 10 \mathrm{~min}$ seguido de 45 ciclos de $94^{\circ} \mathrm{C} / 15 \mathrm{~s} ; 56^{\circ} \mathrm{C} / 25 \mathrm{~s} ; 72^{\circ} \mathrm{C} / 25 \mathrm{~s}$ y una extensión final de $75^{\circ} \mathrm{C} / 5 \mathrm{~min}$.

\section{Detección de rotavirus y adenovirus}

La detección de RV y AdV se realizó en el Laboratorio de Microbiología del HGGB que lleva a cabo rutinariamente el diagnóstico cualitativo de estos virus, utilizando el kit Immunocromatography, VIKIA ${ }^{\circledR}$ Rota-Adeno (Biomerieux) según las instrucciones del fabricante.

Análisis de ficha clínica: El análisis de la ficha clínica de cada paciente se llevó a cabo al finalizar el análisis etiológico de la diarrea viral y se extrajo la siguiente

Tabla 1.Distribución etaria de NoV, RV y AdV en niños hospitalizados con diarrea. Hospital Guillermo Grant Benavente, Concepción Chile. Diciembre 2007-diciembre 2008

\begin{tabular}{|c|c|c|c|c|}
\hline & $\begin{array}{l}\text { Total pacientes } \\
\qquad n=145\end{array}$ & $\begin{array}{c}\text { Lactantes } \\
0-2 \text { años } \\
71 \% \text { (n: 103) }\end{array}$ & $\begin{array}{l}\text { Pre-escolares } \\
2-4 \text { años } \\
11,7 \% \text { (n: 17) }\end{array}$ & $\begin{array}{c}\text { Escolares } \\
\text { 4-14años } \\
17,3 \% \text { (n: } 25)\end{array}$ \\
\hline Norovirus & $25,5 \% \quad(37 / 145)^{*}$ & $34 \%(35 / 103)^{*}$ & $11,8 \%(2 / 17)$ & 0 \\
\hline Rotavirus & $15,9 \% \quad(23 / 145)$ & $17,5 \%(18 / 103)$ & $11,8 \%(2 / 17)$ & $12 \%(3 / 25)$ \\
\hline Adenovirus & $6,2 \% \quad(9 / 145)$ & $7,8 \%(8 / 103)$ & 0 & $4 \%(1 / 25)$ \\
\hline S/etiología de la diarrea & $55,2 \% \quad(76 / 145)$ & $43,7 \%(45 / 103)$ & $82,4 \%(14 / 17)$ & $84 \%(21 / 25)$ \\
\hline
\end{tabular}

información: demografía (edad y sexo), causa de la internación y tiempo de la hospitalización al momento de obtenerla muestra, época del año de la toma de la muestra, características y duración de la diarrea.

Análisis de datos: El análisis estadístico se realizó por test de $\chi^{2}$ de Pearson para la comparación de proporción de infección por NoV, RV y AdV de la población total.

\section{Resultados}

\section{Presencia de NoVy RV/AdV en niños con diarrea y controles}

En 47,6\% de los 145 niños hospitalizados con diarrea se detectó un agente viral, siendo significativamente mayor $(\mathrm{p}<0,05)$ la infección causada por NoV, $(25,5 \%)$, seguida por RV $(15,9 \%)$ y AdV (6,2\%) (Tabla 1$)$. El genogrupo predominante de NoV fue GII en 62,2\% (23/37), seguido de GI 24,3\% (9/37), mientras que 13,5\% (5/37) presentó co-infección con ambos genogrupos. Dos niños tuvieron infección mixta $\mathrm{NoV} / \mathrm{RV}$, uno presentó $\mathrm{NoV} /$ AdV y otro RV/AdV.

Dos de los 57 controles sanos (sin diarrea) presentaron NoV (3,5\%): un niño de 2 años y 4 meses con identificación del geno-grupo GI y una niña de 9 meses con GII.

\section{Distribución etaria de la infección por NoV, $R V y$ AdV}

Los lactantes -que constituían $71 \%$ de los pacientestuvieron infección por NoV con una frecuencia de $34 \%$, significativamente mayor $(\mathrm{p}<0,05)$ que la infección por RV y AdV (Tabla 1). El 65,7\% (23/35) de los NoV encontrados en lactantes correspondían al GII, 22,9\% (8/35) eran GI y $11,4 \%$ presentaba infección GI/GII (4/35). En pre-escolares (2-4 años) hubo 11,8\% de infección, tanto de NoV como de RV, y no se encontró AdV. Los escolares (mayores de 4 años) presentaron $12 \%$ de RV, $4 \%$ de AdV y ausencia de NoV (Tabla 1 ).

\section{Distribución de la infección por NoV, RV y AdV según género}

La proporción de infección por NoV fue significativamente mayor $(p<0,05)$ en varones $(31,5 \%)$ que en niñas $(19,4 \%)$. La infección por RV y AdV fue también mayor en varones, pero la diferencia no fue significativa (Tabla 2). Hubo 69,6\% (16/23) de NoV genogrupo GII en niños y $50 \%$ (7/14) en niñas, mientras que se encontró $17,4 \%$ (4/23) de NoV GI en varones y 35,7\% (5/14) en niñas. Se detectó co-infección por ambos genogrupos en $13 \%$ de los niños y $14,3 \%$ de las niñas. La diferencia de infección por $\mathrm{NoV}$, de acuerdo al género, también fue significativa en lactantes $(\mathrm{p}<0,05)$ (Tabla 3), siendo mayor en varones $(40,7 \%)$ que en niñas $(26,7 \%)$. En cambio en infecciones 
causadas por RV y AdV no existió diferencia de infección según género.

\section{Diarreas virales en niños por causa de hospitalización}

De los 145 niños bajo estudio, 63,4\% (92/145) fueron hospitalizados por gastroenteritis aguda, caracterizado por deposiciones semisólidas o líquidas 2 a 3 veces por día. El 36,6\% restante (53/145) correspondió a niños hospitalizados por otras causas (cuadros respiratorios, leucemia, inmunodeficiencias, infecciones del tracto urinario y otras) y que presentaron un episodio de diarrea entre 3 y 11 días posteriores a su ingreso. Los niños hospitalizados por diarrea presentaron el mismo porcentaje de infección por NoV que por RV (21,7\%) (Tabla 2); en tanto que en niños con infección nosocomial, la proporción de infección por NoV fue mayor $(32,1 \%)$ que por RV $(5,7 \%)$ (Tabla 4). En ambos grupos predominó la presencia del GII.

\section{Presentación estacional de las diarreas virales}

Las diarreas causadas por NoV predominaron durante la mayor parte del año, excepto en verano, cuando hubo más diarreas causadas por RV. La presencia de AdV se mantuvo baja durante todo el año (Figura 1).

\section{Discusión}

El reconocimiento de NoV se ha incrementado en los últimos cinco a 10 años dada la disponibilidad de técnicas más sensibles de detección como la utilizada en este estudio ${ }^{30,31}$. Actualmente se considera que $\mathrm{NoV}$ sería causa de hospitalización por gastroenteritis en niños de todo el mundo, con prevalencia e impacto clínico cercano al rango de lo observado para $\mathrm{RV}^{30-32}$. Varios estudios han señalado que NoV es responsable de 40 a $50 \%$ de los brotes de diarrea esporádicas agudas en personas de cualquier edad, en países desarrollados ${ }^{10,33-35}$. En la actualidad, NoV es una de las causas más frecuentes de gastroenteritis esporádica viral aguda en el mundo ${ }^{33-35}$. El CDC de E.U.A., ha reportado que $\mathrm{NoV}$ es responsable de aproximadamente $96 \%$ de todos los brotes de gastroenteritis viral secundarios a infección alimentaria y que causa más de 23 millones de infecciones anuales en E.U.A. ${ }^{36}$. Esta situación epidémica se asocia especialmente a aglomeraciones humanas como ocurre en cruceros, escuelas, hospitales, campamentos militares y otros $^{5-7}$.

En nuestro estudio, la etiología viral por NoV en diarrea fue de $25,5 \%$ para el total de pacientes y de $34 \%$ para los niños bajo 2 años de edad, valores significativamente superiores a los obtenidos para RV $(15,9$ y $17,5 \%$, respectivamente). La información aportada por el MINSAL para el 2011 fue similar (NoV 27,1 y RV 19,6\%) ${ }^{17}$.

La frecuencia de NoV en los centros centinela durante
Tabla 2. Distribución de las diarreas causadas por NoV, RV y AdV en el total de niños según género. Hospital Guillermo Grant Benavente, Concepción Chile. Diciembre 2007-diciembre 2008

\begin{tabular}{|lcc|}
\hline Etiología de la diarrea & $\begin{array}{c}\text { Hombre } \\
\mathbf{5 0 , 3} \%(\mathbf{n}: \mathbf{7 3})\end{array}$ & $\begin{array}{c}\text { Mujeres } \\
\mathbf{4 9 , 7 \%}(\mathbf{n : ~ 7 2 )}\end{array}$ \\
Norovirus & $31,5 \%(23 / 73)^{*}$ & $19,4 \%(14 / 72)$ \\
Rotavirus & $16,4 \%(12 / 73)$ & $15,3 \%(11 / 72)$ \\
Adenovirus & $8,2 \% \quad(6 / 73)$ & $4,2 \% \quad(3 / 72)$ \\
S/. etiología de la diarrea & $46,6 \%(34 / 73)$ & $63,9 \%(46 / 72)$ \\
\hline *Valor estadísticamente significativo $(p<0,05)$ de NoV entre hombre y mujer. \\
\hline
\end{tabular}

Tabla 3. Proporción de infección por Nov, RV y AdV en lactantes según género. Hospital Guillermo Grant Benavente, Concepción Chile. Diciembre 2007-diciembre 2008

\begin{tabular}{|lccc|} 
& \multicolumn{1}{c}{ Hombres } \\
$\mathbf{n = 5 4}(\mathbf{5 2 , 4} \%)$ & $\mathbf{n}=\mathbf{4 9}(\mathbf{4 7}, \mathbf{6 \%})$ & $\mathbf{p}$ \\
Norovirus & $40,7 \%(22 / 54)$ & $26,5 \%(13 / 49)$ & $<0,05$ \\
Rotavirus & $16,7 \%(9 / 54)$ & $18,4 \%(9 / 49)$ & $\mathrm{ns}$ \\
Adenovirus & $9,3 \%(5 / 54)$ & $6,1 \%(3 / 49)$ & $\mathrm{ns}$ \\
S/infec. viral & $37 \%(20 / 54)$ & $51 \%(25 / 49)$ & \\
\hline
\end{tabular}

Tabla 4. Distribución de las diarreas causadas por NoV, RV y AdV en el total de niños según causa de hospitalización

\begin{tabular}{lcc|} 
& $\begin{array}{c}\text { Hospitalizado por diarrea } \\
\mathbf{6 3 , 4 \%}(\mathbf{n}=\mathbf{9 2})\end{array}$ & $\begin{array}{c}\text { Hospitalizado por otras causas } \\
\mathbf{3 6 , 6 \%} \mathbf{( n = 5 3 )}\end{array}$ \\
Norovirus & $21,7 \%(20 / 92)$ & $32,1 \%(17 / 53)$ \\
Rotavirus & $21,7 \%(20 / 92)$ & $5,7 \% \quad(3 / 53)$ \\
Adenovirus & $7,6 \%(7 / 92)$ & $3,8 \%(2 / 53)$ \\
S/. etiología de la diarrea & $52,2 \%(48 / 92)$ & $60,4 \%(32 / 53)$ \\
\hline
\end{tabular}

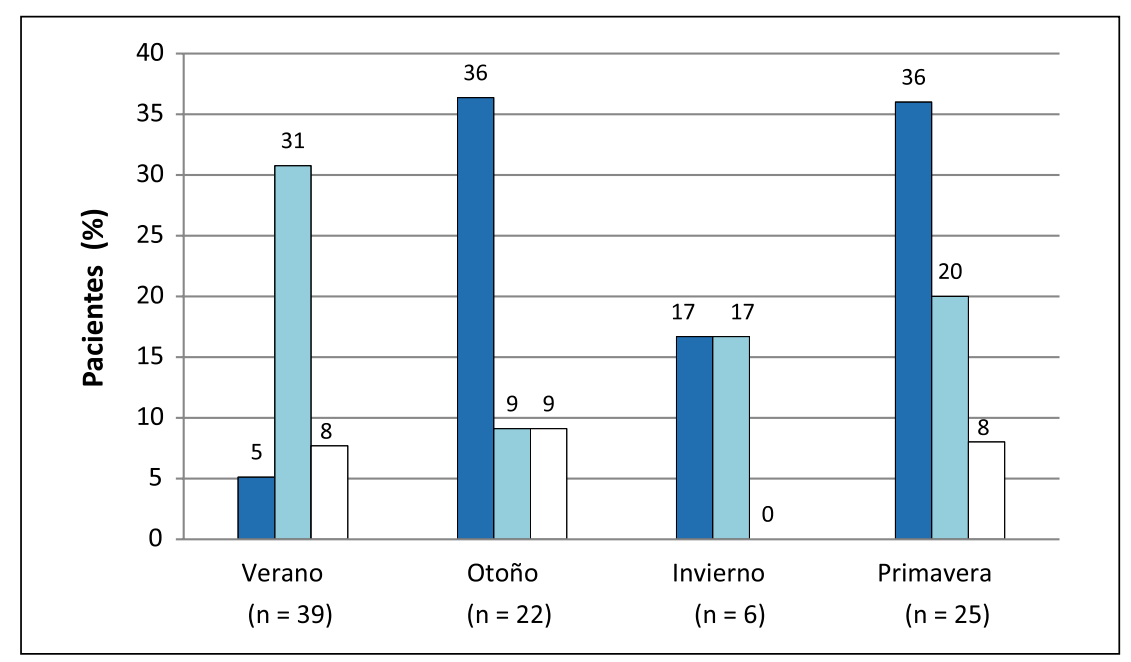

Figura 1. Presentación estacional de diarreas virales (NoV, RV y AdV) en niños hospitalizados. Barras azules $(\square)$ : presencia de NoV; Barras celeste $(\square)$ : presencia de RV; Barra blanca ( $\square$ ): presencia de AdV. 
el año 2012 continuó siendo alta en lactantes, especialmente en las ciudades de Antofagasta, Valparaíso y Santiago con prevalencia de NoV en las dos últimas ${ }^{24}$.

Estos valores confirman la tendencia actual de altas frecuencias de infección por NoV en niños bajo 2 años de edad; así se han publicado frecuencias de:33,4\% en Brasil, 24,0\% en Argentina, 21,3\% en Perú y 13,0\% en Venezuela ${ }^{37-39}$. En nuestro estudio, encontramos una diferencia significativa en la infección por NoV del GII, en relación al género del paciente, con mayor frecuencia de infección en varones que en niñas, característica que también fue observada en estudios de diarreas agudas en niños de Venezuela y Colombia, con mayor incidencia en varones ${ }^{40,41}$. Aún no se conoce la causa de la mayor propensión a la infección por NoV según el género del paciente.

Observamos también que en más de $50 \%$ de los niños no hubo diagnóstico etiológico de diarrea; para ello se revisaron los resultados de los exámenes de coprocultivo y exámenes parasitológicos seriados de deposición, realizado en el laboratorio de microbiología del HGGB. Este hallazgo ha sido comunicado también en otros trabajos, en que alrededor de 40 a $50 \%$ de los casos permanecen sin un diagnóstico causal $^{40,42}$. La falta de diagnóstico etiológico de diarreas es frecuente, de ahí la importancia de incorporar técnicas diagnósticas más sensibles ya que los tests de rutina generalmente resultan negativos ${ }^{2,7,8}$.

La detección de NoV es difícil debido a la gran variabilidad genética que dificulta el diagnóstico de laboratorio de rutina; la técnica de ELISA tiene una baja sensibilidad que obliga a una confirmación de un resultado negativo por métodos moleculares ${ }^{32,41,43}$. La introducción del diagnóstico empleando RPC en tiempo real ha sido de gran importancia para mejorar el diagnóstico y establecer el rol relevante de NoV en brotes epidémicos agudos y en casos esporádicos de diarrea. Este método diagnóstico ha permitido demostrar la amplia heterogeneidad y rápida evolución de las variantes genéticas de $\mathrm{NoV}^{8,9,11-15}$; La diversidad antigénica y la falta de sistema de cultivo in vitro del virus mantendrá al diagnóstico molecular como el mejor método de detección de NoV. La RPC a tiempo real ha emergido como el estándar para el diagnóstico y detección de NoV, ya que combina un alto rendimiento, reproducibilidad, sensibilidad y especificidad ${ }^{11,27,42-46}$.

El 36,6\% de los pacientes en este estudio fueron ingresados al hospital con un diagnóstico diferente al de una gastroenteritis, adquiriendo la infección probablemente en forma nosocomial (plazo de latencia para los síntomas de 3 o más días), siendo mayor para $\operatorname{NoV}(32,1 \%)$ que para RV (5,7\%). Esto se debería a la resistencia y amplia transmisibilidad de NoV a través de contacto persona a persona, vía fecal-oral, consumo de alimentos o agua contaminados $^{8-11,13}$, lo que favorece su propagación en recintos semi-confinados como hospitales ${ }^{47,48}$. Además, la trasmisión se facilita porque la dosis infectante de NoV es muy baja, alrededor de 10 partículas virales; la excreción viral es intensa y persiste por varias semanas aún después de desaparecidos los síntomas; posee gran estabilidad ambiental, ya que resiste cambios de temperatura, $\mathrm{pH}$ ácidos y desinfectantes como cloro y etanol, permitiendo múltiples re-infecciones ${ }^{5,8-11,13}$. Se ha demostrado que la contaminación ambiental con NoV se asocia frecuentemente al mayor tráfico en la unidad de pacientes hospitalizados y que la mejor manera de controlar este problema es mantener estrictos hábitos de higiene, especialmente lavado de manos, utilizando gel de manos con alcohol ${ }^{25,26}$.

La variación estacional de la presentación de brotes de NoV está reconocida, ocurriendo generalmente en invierno y otoño ${ }^{10,11}$. Una posible explicación de la baja presentación de NoV en verano podría ser la limitada circulación de virus en esta época del año, con larga sobrevivencia en la población hospedera y poca reactivación estacional $^{49}$. Se piensa que los factores asociados a la estacionalidad del NoV se deberían a una combinación de factores ambientales y de comportamiento que regularían la transmisión, virulencia y persistencia de los viriones en la población hospedera ${ }^{49}$.

Nuestros hallazgos demuestran que NoV es un agente frecuente en niños y, que debe considerarse en el diagnóstico etiológico de pacientes admitidos con SDA, especialmente en la población pediátrica, donde se reportan cada vez mayores tasas de infección, encontrándose una elevada prevalencia dependiendo de la edad, lo que sugiere una exposición temprana y repetida ${ }^{32,38,50}$. Por lo anterior, tendrá cada vez mayor importancia realizar estudios de prevalencia y ahondar en el conocimiento del significado clínico en niños con gastroenteritis aguda y así ayudar en la prevención y control de estos virus s,5,29,50 $^{1}$.

En conclusión, los NoV aparecen en nuestra región como una importante etiología de diarreas virales que incluso superó la presentación por RV en el presente estudio.

\section{Proyecciones}

Nuevos estudios podrán determinar las fuentes de infección ambiental, variabilidad genética y características epidemiológicas de los NoV para establecer estrategias de prevención e intervención.

\section{Resumen}

Introducción: Los norovirus ( $\mathrm{NoV}$ ) son virus ARN altamente contagiosos, resistentes, variables genéticamente y una de las etiologías más frecuente de gastroenteritis viral esporádica mundial. Este es el primer trabajo en 
Concepción, Chile, de búsqueda de NoV como etiología viral de diarreas en niños hospitalizados. Objetivo: Determinar la presencia y genogrupo de NoV en niños con diarrea y compararla con la frecuencia de rotavirus (RV) y adenovirus (AdV). Material y Método: Estudio descriptivo, prospectivo de un año, en niños de 0-14 años ingresados por diarrea aguda o que la adquirieron dentro del hospital. La muestra de deposiciones diarreica se tomó una sola vez por paciente. Las fichas clínicas se analizaron al finalizar el estudio etiológico. Para la detección de NoV se utilizó RPC-TR a en tiempo real con sondas Taqman ${ }^{\circledR}$ y para detección de $\mathrm{RV} / \mathrm{AdV}$, el kit VIKIA ${ }^{\circledR}$ de inmunocromatografia. Resultados: La infección por NoV $(25,5 \%)$ fue significativamente más frecuente que por RV $(15,9 \%)$ y $\mathrm{AdV}(6,2 \%)$. La mayor presencia de infección fue en pacientes bajo2 años de edad (n: 103): NoV 34,0\%, RV 17,5\%, AdV 7,8\%. La detección en niños hospitalizados por diarrea fue: NoV y RV 21,7\% cada uno; AdV 7,6\%. En niños con diarrea nosocomial hospitalizados por otras causas se detectó NoV en $32,1 \%$, RV en 5,7\% y AdV en 3,8\%. La presencia de NoV fue significativamente mayor en varones $(31,5 \%)$ que en niñas $(19,4 \%)$. El promedio de diarreas durante el año fue mayor para $\operatorname{NoV}(30,3 \%)$ que para $\operatorname{RV}(14,7 \%)$, excepto en verano. Discusión y Conclusión: La presencia de NoV fue mayor que la de RoV en niños con diarrea y con una tendencia nosocomial que podría deberse a las características del virus que favorece infecciones de ambiente confinado, como hospitales, asilos y cruceros. La infección por NoV presentó características definidas, en edad, género, ocurrencia estacional y relevancia nosocomial, que aportan datos epidemiológicos importantes.

\section{Referencias bibliográficas}

1.- Dennehy P H. Acute diarrheal disease in children: epidemiology, prevention, and treatment. Infect Dis Clin North Am 2005; 19 (3): 585-602.

2.- O’Ryan M, Prado V, Pickering L K. A millennium update on pediatric diarrheal illness in the developing world. Semin Pediatr Infect Dis 2005 ; 16: 125-36.

3.- De Caldas G. Gastroenteritis de etiología viral no causadas por rotavirus. En: Vox Paediatrica 2007; 15 (1).

4.- Clark B, Mckendrick M. A review of viral gastroenteritis. Curr Opin Infect Dis 2004; 17: 461-9.

5.- Glass Ri, Bresee J, Jiang B, Gentsch J, Ando T, Fankhauser R, et al. Gastroenteritis viruses: an overview. Novartis Found Symp 2001; 238: 5-25.

6.- Lopman B, Reacher M, Vipond I, Sarangi J, Brown D. Clinical manifestation of norovirus gastroenteritis in healthcare settings. Clin Infect Dis 2004; 39: 318-24.

7.- Atmar R, Estes M. The epidemiologic and clinical importance of norovirus infection. Gastroenterol Clin North Am 2006; 35: 275-90.

8.- Bon F, Ambert-Balay K, Giraudon H, Kaplon J, Le Guyader S, Pommepuy M, et al. Molecular epidemiology of caliciviruses detected in sporadic and outbreak cases of gastroenteritis in France from December 1998 to February 2004. J Clin Microbiol 2005; 43: 4659-64.

9.- Bull R, Mciver C, Rawlinson W, White P. Emergence of a new norovirus genotype II.4 variant associated with global outbreaks of gastroenteritis. J Clin Microbiol 2006; 44: 327-33.

10.- Karst S. Pathogenesis of noroviruses, emerging RNA viruses. Viruses 2010; 2: 748-81.
11.- Glass R, Parashar U, Estes M. Norovirus gastroenteritis. N Engl J Med 2009; 361: 1776-85.

12.- Lopman B, Reacher M, Vipond I, Hill D, Perry C, Halladay T, et al. Epidemiology and cost of nosocomial gastroenteritis, Avon, England, 2002-2003. Emerg Infect Dis 2004; 10: $1827-34$.

13.- Simpson R, Aliyu S, Iturriza-Gómara $\mathrm{K}$, Desselberger U, Gray J. Infantile viral gastroenteritis: on the way to closing the diagnostic gap. J Med Virol. 2003; 70: 258-62.

14.- Zheng D, Ando T, Fankhauser R, Beard S, Glass R, Monroe S. Norovirus classification and proposed strain nomenclature. Virol 2006; 346 : 312-23.

15.- Vinje J, Hamidjaja R, Sobsey M. Development and application of a capsid VP1 (region D) based reverse transcription PCR assay for genotyping of genogroup I and II noroviruses. J Virol Methods 2004; 116: 109-17.

16.- Díaz T J. Diarreas en menores de 5 años. El Vigía 24, Boletín de Vigilancia en Salud Pública 2007; 10 (24): 10-12.

17.- Dunner A. Diarreas agudas, Situación epidemiológica. Semanas 1 a 52 año 2011. Departamento de Epidemiología, Ministerio de Salud, Gobierno de Chile. http://epi.minsal.cl/ epi/html/AtlasInteractivos/AB_101/Diarrea.htm. [Consultado 26 junio 2012].

18.- O'Ryan M, Pérez-Schael I, Mamani N, Peña A, Salinas B, González G, et al. Rotavirus-associated medical visits and hospitalization in South America: a prospective study at three large sentinel hospitals. Pediatr Infect Dis J 2001; 20 (7): 685-93.

19.- O'Ryan, Vial P, Mamani N, Jiang X, Estes M, Ferrecio C, et al. Seroprevalence of Norwalk virus and Mexico virus in children individuals: assessment of independent risk factors for antibody acquisition. Clin Infect Dis 1998; 27 : 789-95.

20.- O’Ryan M, Mamani N, Gaggero A, Avendaño L, Prieto S, Pena A, et al. Human caliciviruses are a significant pathogen of acute sporadic diarrhea in children of Santiago. J Infect Dis 2000; 182: 1519-22.

21.- Vidal R, Solari V, Mamani N, Jiang X, Vollaire J, Roessler P, et al. Caliciviruses and foodborne gastroenteritis, Chile. Emerg Infect Dis 2005; 11: 1134-7.

22.- O’Ryan M, Peña A, Vergara R, Díaz J, Mamani N, Cortés H, et al. Prospective characterization of norovirus compared with rotavirus acute diarrhea episodes in Chilean children. Pediatr Infect Dis J 2010; 29 (9): 855-9.

23.- Cachicas V, Villarroel O. Detección de norovirus en muestras de agua de la ciudad de Antofagasta-Chile. Año 2010. Gobierno de Chile, Instituto de Salud Pública, Subdepartamento de Alimentos y Nutrición 2010

24.- Poulain C. Diarreas Agudas, Situación epidemiológica. Semanas 1 a 19 año 2012. Atlas-BEM 104, Departamento de Epidemiología, Ministerio de Salud, Gobierno de Chile. http://epi.minsal.cl [Consultado 25 febrero 2013].

25.- Hansen S, Stamm-Balderjahn S, Zuschneid I, Behnke M, Ruden H, Vonberg R, et al. Closure of medical departments during nosocomial outbreaks: data from a systematic analysis of the literature. J. Hosp Infect 2007; 65: 348-53.

26.- Weber DJ, Rutala WA, Miller MB, Huslage K, Sickbert-Bennett E. Role of hospital surfaces in the transmission of emerging health care associated pathogens: Norovirus, Clostridium difficile, and Acinetobacter species. Am J Infect Control 2010; 38: S25-33.

27.- Trujillo A, Mccaustland K, Zheng D, 
Hadley L, Vaughn G, Adams S, et al. Use of TaqMan real-time reverse transcription-PCR for rapid detection, quantification, and typing of norovirus. J Clin Microbiol 2006; 4 (4): 1405-12.

28.- Apaza S, Espetia S, Gilman Rh, Montenegro S, Pineda S, Herhold F, et al. Detection and genogrouping of noroviruses from children's stools by Taqman one-step RT-PCR. J Vis Exp 2012; (65), e3232, DOI: 10.3791/3232.

29.- Kageyama T, Kojima S, Shinohara M, Uchida K, Fukushi S, Hoshino FB, et al. Broadly reactive and highly sensitive assay for norwalk-like viruses based on real-time quantitative reverse transcription-PCR. J Clin Microbiol 2003; 41: 1548-57.

30.- Koo H, Ajami N, Atmar R, Dupont H. Noroviruses: The principal cause of foodborne disease. Discov Med 2010; 10 (50): 61-70.

31.- Blanton L, Adams S, Beard R, Wei G, Bulens S, Widdowson M, et al. Molecular and epidemiologic trends of caliciviruses associated with outbreaks of acute gastroenteritis in the United States, 2000-2004. J Infect Dis 2006; 193: 413-21.

32.- Bucardo F, Nordgren J, Carlsson B, Paniagua M, Lindgren P, Espinoza F. Pediatric norovirus diarrhea in Nicaragua. J Clin Microbiol 2008; 46: 2573-80.

33.- Musher D, Musher B. Contagious acute gastrointestinal infections. N Eng J Med 2004; 351: $2417-28$

34.- Turcios R, Widdowson M, Sulka A, Mead P, Glass R. Reevaluation of epidemiological criteria for identifying outbreaks of acute gastroenteritis due to norovirus: United States, 1998-2000. Clin Infect Dis 2006; 42: 964-9.
35.- Svraka S, Duizer E, Vennema H, De Bruin E, Van Der Veer B, Dorresteijn B, et al. Etiological role of viruses in outbreaks of acute gastroenteritis in The Netherlands from 1994 through 2005. J Clin Microbiol 2007; 45: 1389-94.

36.- Maccannell T, Umscheid C, Agarwal R, Lee I, Kuntz G, Stevenson K, et al. Guideline for the prevention and control of norovirus gastroenteritis outbreaks in healthcare settings. CDC 2011; 1-52 http://www.cdc.gov/hicpac/ pdf/norovirus/Norovirus-Guideline-2011.pdf [Consultado 28 febrero 2013]

37.- Ferreira M S, Xavier M, Tinga A C, Rose T L, Fumian T M, Fialho A M, et al. Assessment of gastro-enteric viruses frequency in a children's day care center in Rio de Janeiro, Brazil: a fifteen year study (1994-2008). PLoS ONE 2012; 7 (3): e33754.

38.- Bereciartu A, Bok K, Gómez J. Identification of viral agents causing gastroenteritis among children in Buenos Aires, Argentina. J Clin Virol 2002; 25:197-203

39.- Yori P P, Schwab K, Gilman R H, Nappier S, Portocarrero D V, Black R E, et al. Norovirus highly prevalent cause of endemic acute diarrhea in children in the Peruvian Amazon. Pediatr Infect Dis J 2009; 28: 844-7.

40.- Gonzalez G, Liprandi F, Ludert J. Molecular epidemiology of enteric viruses in children with sporadic gastroenteritis in Valencia, Venezuela. $\mathrm{J}$ Med Virol 2011; 83: 1972-82.

41.- Gutiérrez M F, Urbina D, Matiz A, Puello M, Mercado M, Parra M, et al. Comportamiento de la diarrea causada por virus y bacterias en regiones cercanas a la zona ecuatorial. Colomb Med 2005; 36: 6-14.

42.- Ribes J, Buesa J. Infecciones por norovirus.
Enferm Infecc Microbiol Clin 2010; 28: 51-5.

43.- De Cal W, Revilla A, Del Alamo J, Roman E, Moreno S, Sánchez-Fauquier A. Evaluation of two commercial enzyme immunoassays for the detection of norovirus in faecal samples from hospitalized children with sporadic acute gastroenteritis. Clin Microbiol Infect 2007; 13: 341-3.

44.- Dreier J, Stormer M, Made D, Burkhardt S, Kleesiek K. Enhanced reverse transcriptionPCR assay for detection of norovirus genogroup I. J Clin Microbiol 2006; 44: 2714-20.

45.- Scipioni A, Bourgot I, Mauroy A, Ziant D, Saegerman C, Daube G, et al. Detection and quantification of human and bovin noroviruses by a TaqMan RT-PCR assay with a control for inhibition. Mol Cell Probes 2008; 4: 215-22.

46.- Montes M, Iturriza-Gómara M. Molecular methods for the diagnosis of acute viral gastroenteritis. Enferm Infecc Microbiol Clin 2008; 26 (9): 81-5.

47.- Rosenfeldt V, Vesikari T, Pang X L, Zeng S Q, Tvede M, Paerregaard A. Viral etiology and incidence of acute gastroenteritis in young children attending day-care centers. Pediatr Infect Dis J 2005; 24: 962-5.

48.- Rhinehart E, Walker S, Murphy D, O'Reilly K, Leeman P. Frequency of outbreak investigations in US hospitals: Results of a national survey of infection preventionists. Am J Infect Control 2012; 40: 2-8.

49.- Rohayem J. Norovirus seasonality and the potential impact of climate change. Clin. Microbiol. Infect.2009; 15: 524-7.

50.- Koopmans, Marion. Progress in understanding norovirus epidemiology. Curr Opin Infect Dis. In. Gastrointestinal infections: Ed. Beeching NJ\& Clinton AW 2008; 21: 544-52. 